\title{
Effect of Cut-Off Frequency of Butterworth Filter on Detectability and Contrast of Hot and Cold Regions in Tc-99m SPECT
}

\author{
Inayatullah Shah Sayed*, Nor Syahirah Mohamed Nasrudin \\ Department of Diagnostic Imaging and Radiotherapy, Kulliyyah (Faculty) of Allied Health Sciences, \\ International Islamic University of Malaysia, Kuantan, Malaysia \\ Email: "inayatullah@iium.edu.my
}

Received 30 January 2016; accepted 8 February 2016; published 29 February 2016

Copyright (C) 2016 by authors and Scientific Research Publishing Inc.

This work is licensed under the Creative Commons Attribution International License (CC BY). http://creativecommons.org/licenses/by/4.0/

(c) (7) Open Access

\begin{abstract}
In SPECT, noise is one of the major limitations that degrade image quality. To suppress the noisy signals in an image, digital filters are most commonly applied. However, in SPECT image reconstruction, selection of an appropriate filter and its functions has always remained a difficult task. In this work an attempt was made to investigate the effects of varying cut-off frequencies and in keeping the order of Butterworth filter constant on detectability and contrast of hot and cold regions images. A new insert simulating hot and cold regions which provides similar views in a reconstructed image was placed in the phantom's cylindrical source tank and imaged. Tc-99m radionuclide was distributed uniformly in the phantom. SPECT data were collected in a $20 \%$ energy window centered at $140 \mathrm{keV}$ by a Philips ADAC Forte dual head gamma camera mounted with a LEHR collimator. Images were generated by using the filtered backprojection technique. A Butterworth filter of order 5 with cut-off frequencies 0.35 and 0.45 cycles $\cdot \mathrm{cm}^{-1}$ was applied. Images were examined in terms of hot and cold regions, detectability and contrast. Results show that the hot and cold regions' detectability and contrast vary with the change of cut-off frequency. With a 0.45 cycles $\cdot \mathbf{c m}^{-1}$ cut-off frequency, a significant enhancement in contrast of cold regions was achieved as compared to a 0.35 cycles $\cdot \mathrm{cm}^{-1}$ cut-off frequency. Furthermore, the detectability of hot and cold regions improved with the use of a $0.45 \mathrm{cycles} \cdot \mathrm{cm}^{-1}$ cut-off frequency. In conclusion, image quality of hot and cold regions affected in a different way with a change of cut-off frequency. Thus, care should be taken in selecting the filter cut-off frequency prior to reconstruction of images; particularly, when both types of regions are expected in the reconstructed image.
\end{abstract}

\section{Keywords}

Detectability, SPECT, Noise, Image Quality, Cut-Off Frequency, Mathematical Filters

\footnotetext{
${ }^{*}$ Corresponding author.
}

How to cite this paper: I.S. Sayed and N.S.M. Nasrudin (2016) Effect of Cut-Off Frequency of Butterworth Filter on Detectability and Contrast of Hot and Cold Regions in Tc-99m SPECT. International Journal of Medical Physics, Clinical Engineering and Radiation Oncology, 5, 100-109. http://dx.doi.org/10.4236/ijmpcero.2016.51011 


\section{Introduction}

In clinical single photon emission computed tomography (SPECT), the quality of an image plays a significant role in diagnosing abnormalities and in the proper management of patients. However, the image quality is limited by a number of reasons; among them is noise [1]-[5]. Therefore, it is essential to carefully tackle this issue. Usually SPECT images are reconstructed using filtered back projection and iterative techniques where the filtering is an important part of the image reconstruction process for addressing noisy signals which affect the quality of images to a greater extent [6]. In this regard, a variety of mathematical filters have been applied for various purposes, e.g., removal of star artifacts, statistical noise reduction, signal enhancement and restoration of image resolution. Generally, filters are categorized as high pass and low pass frequency filters. High pass filter, e.g., ramp filters do not allow low frequencies resulting in the rise of blurring effects in image. These filters sharpen the edges of images, but, amplify the statistical noise present in the raw data of the image [7]. In order to overcome the problem of amplification of high frequencies, a ramp filter is combined with the low pass filters. Low pass filters suppress high frequency signals and permit low frequency signals which result in smoothness in the reconstructed image. Hence, these filters are also known as smoothing filters. The disadvantage of low pass filters is a decrease in image contrast.

Furthermore, low pass filters are described by two functions, e.g., cut-off frequency and order. Cut-off frequency is the frequency above which the noise is removed. The image noise, resolution, and overall image quality are affected by the value of the cut-off frequency which represents the maximum frequency the filter will allow. Adjustment of the high cut-off frequency improves the spatial resolution but the image appears noisier as compared to the selection of a low cut-off frequency. In contrast, a low cut-off frequency provides a smoother image at the cost of contrast. The cut-off frequency roll off is controlled by the order of the filter which characterizes the steepness of the roll off. A higher order results in a sharper fall. However, both functions have a significant role to play in achieving a better quality image [5]-[7].

These filters are basically mathematical functions applied to an image's matrix in the frequency domain. With mathematical filters many of noisy signals are removed and true signals are retained within the range of the frequency [8]. When they are applied to the data, some useful signals whose frequencies match the noisy signals are also suppressed. Thus, choosing an appropriate cut-off frequency and filter order to achieve optimum filtration is a difficult task [2] [9] [10].

SPECT clinicians' decision making ability to diagnose diseases accurately, providing best possible care and proper management of patients are supplemented by the best quality reconstructed images. Achievement of optimum quality images depends upon many factors, e.g. accurate processing of the image raw data [11]. SPECT data processing or reconstruction of images employs the filtration of noisy signals which is one of the main problems that this imaging modality inherits. The noise is reduced or suppressed by a number of mathematical filters. Application of these filters on the SPECT raw data recover the details of scanned objects in reconstructed images. It is always wished by the clinicians to achieve an ideal image or true duplicate copy of the object/organ under clinical investigations in order to enhance diagnostic accuracy and specificity. Unfortunately, it is impossible to retrieve all the information about the three dimensional objects/organs with the most commonly applied mathematical filters (e.g. Butterworth filter) applied in conjunction with image reconstruction techniques (e.g. Filtered back projection). Literature shows that there is no single universally accepted filter with its constant parameters (cut-off frequency and order) for the use in all clinical SPECT studies. Manufacturers supply a number of filters with their functions as default with all SPECT imaging systems. However, in practice one type of filter with a fixed cut-off frequency and order (particularly cut-off frequency) cannot be used for patient studies, such as, cardiac, liver, bone, brain, etc. [6].

As a matter of fact the accuracy of the results of mathematical filters is affected by the type of object/organ, size, the type of defect (hot and/or cold) \& size and count density in the image data. SPECT images suffer from low count density and non-uniform spatial distribution of radioactive material. Thus, application of single cut-off frequency filter to the data of different types of regions (hot and/or cold) with various sizes would show an enormous impact on the detectability, shape, contrast and resolution in the final reconstructed image. Keeping in view, the change in image quality by varying cut-off frequency of Butterworth filter, reconstructed images of a new hot and cold regions insert (explained in Section 2.1) was studied. Hot and cold regions insert was constructed in such a way, that gamma camera can view both types of regions in a similar fashion from each angle of data acquisition. Thus, the design facilitates an easy and fair comparison of both types of images in terms of 
any effect of the change in filter parameters.

This work investigates the effects of a cut-off frequency of the Butterworth filter on the quality of hot and cold regions image pairs (a pair of hot and cold region (each) of same size) of different diameter with similar transverse views. The filter has been studied in terms of varying cut-off frequency values while keeping the order constant, considering the cut-off frequency is very important and the influence of the order chosen for the image quality is less significant [6]-[8] [12]. The reason to the Butterworth filter frequency $\left(0.35 \mathrm{cycles} \cdot \mathrm{cm}^{-1}\right)$ was chosen for this research was that it is widely used for almost all clinical SPECT studies with order 5 in the department where the study was conducted. Also, in this work, we have selected another cut-off frequency value $\left(0.45\right.$ cycles $\left.\cdot \mathrm{cm}^{-1}\right)$ to determine the effects of changes to cut-off frequency on the hot and cold regions' detectability and contrast. The Butterworth filter in the frequency domain is described by Equation (1).

$$
\text { Butterworth }=\frac{1}{1+\left(\frac{f}{f_{c}}\right)^{2 n}}
$$

where $f_{c}$ is the cut off frequency and $n$ is the order of the filter.

\section{Materials and Methods}

\subsection{SPECT Data Collection}

A Philips ADAC forte dual head gamma camera was used with Pegasys software installed for data collection, reconstruction of image, display and analysis. A new insert (as shown in Figure 1) of hot and cold regions for emission computed tomography (ECT) resolution test phantom was scanned [13]. The insert was placed into the cylindrical source tank. It comprises various sized pairs of hot regions (circular) of different diameters 30.1, 22.6, 18.1, 14.5, 11.6, 9.4, 7.5, 6.1 and $4.9 \mathrm{~mm}$ (in a cold background) holes drilled in a solid acrylic (transparent tissue equivalent density) block of $70 \mathrm{~mm}$ thickness similar to hot regions insert of R. A. Carlson's phantom as shown in Figure 1(a). Another part of the insert consists of cold regions' of various diameters, e.g., 30.0, 22.5, 18.0, 14.4, 11.5, 9.3, 7.4, 6.0, and $4.8 \mathrm{~mm}$ (circular shaped regions in a hot background) at different locations within the phantom as shown in Figure 1(b). There is approximately $+/-0.4 \%$ relative error in each of the hot and cold region pairs of the same diameter. Cold regions' insert was designed by using solid cylindrical acrylic rods $70 \mathrm{~mm}$ long (tissue equivalent density). The cold regions arrangement is similar to the hot regions of R. A. Carlson's phantom i.e., "V" shape with additional pair of $30.1 \mathrm{~mm}$ diameter. The outer diameter of the insert

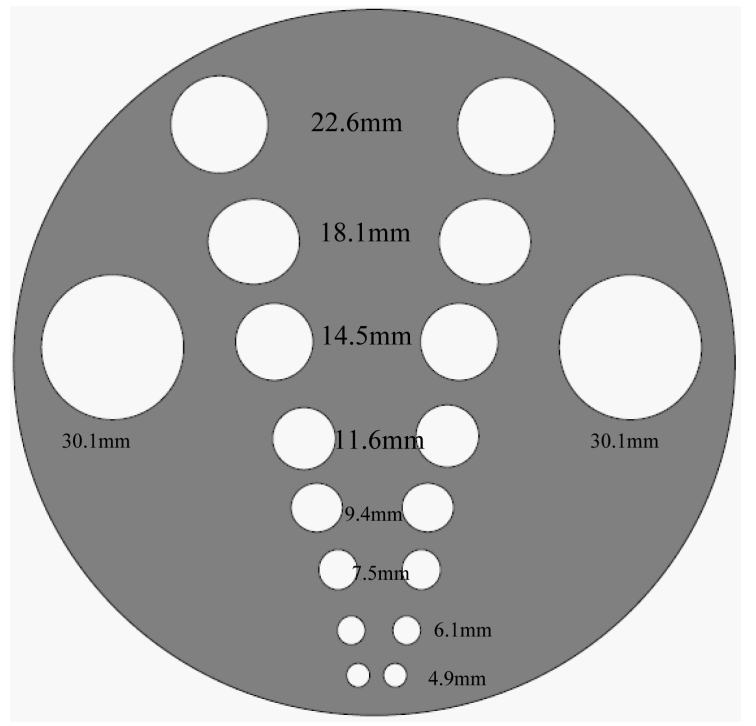

(a)

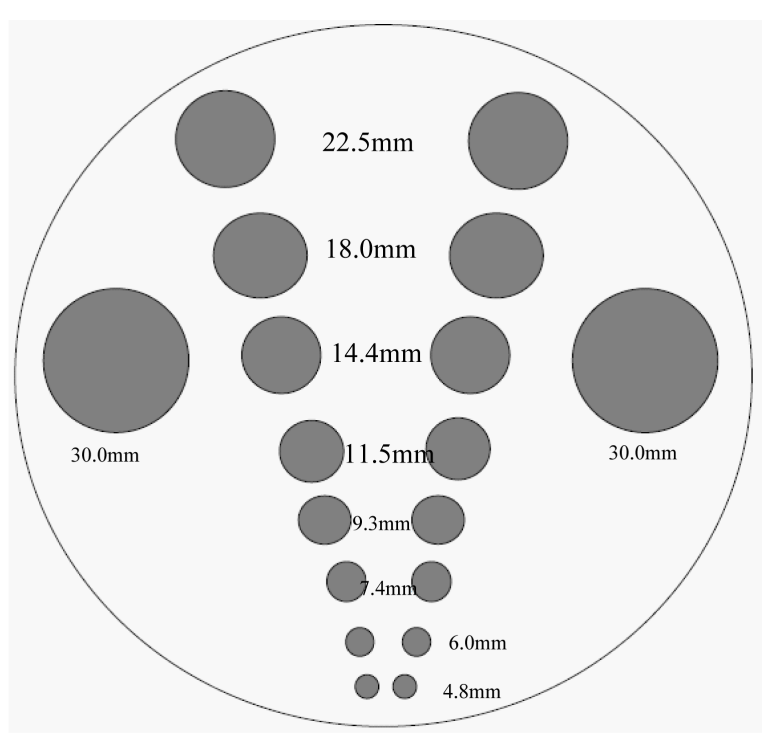

(b)

Figure 1. Schematic cross-sectional views of (a) hot regions insert, and (b) cold regions insert. 
is $208 \mathrm{~mm}$. Base plate-acrylic (5 mm thick) attached with hot regions block with four acrylic pins $5 \mathrm{~mm}$ long and $10 \mathrm{~mm}$ diameter between the hot and cold regions blocks for water/radioactive solution to pass through and distribute properly throughout the tank. The other end is attached with an acrylic plate $(5 \mathrm{~mm}$ thick) to hold the acrylic rods. It should be noted that, the advantage of this new insert is that it provides similar transverse views of both types of regions (hot and cold) in the reconstructed image. Thus, images obtained by scanning this phantom by using different filter functions can easily be analyzed and compared.

The phantom tank was filled with saline water and was left overnight so air bubbles could dissolve. A solution of Tc-99m $(20 \mathrm{mCi})$ was carefully injected into the tank and was left for some time to make sure that the radionuclide was evenly distributed throughout the water before the data collection. The phantom was positioned on the scanning table with its long axis parallel to the collimator and in the centre of the field of view (FOV) of gamma camera. Data were collected by installing a low-energy high resolution (LEHR) collimator in a $128 \times$ 128 matrix. A symmetrical standard energy window was adjusted $(20 \%$ centered at $140 \mathrm{keV})$. Ninety projections (20 seconds per projection) were selected to acquire the data over 360 degrees.

\subsection{SPECT Image Reconstruction}

The transverse images of hot and cold regions were produced using a Filtered back projection (FBP) image reconstruction technique which is fast, easy to implement and most widely applied in SPECT [14]. Chang's attenuation correction technique was applied for compensation of the absorption of gamma photons within the phantom. Data were not corrected for scattered photons therefore; a linear attenuation coefficient value of $0.11 / \mathrm{cm}$ [15] was selected instead of $0.15 / \mathrm{cm}$ for water for $140 \mathrm{keV}$ gamma photons energy. The raw data were corrected for uniformity and center of rotation (COR). Two cut off frequency values 0.35 and $0.45 \mathrm{cycles} \cdot \mathrm{cm}^{-1}$ of order 5 were selected during the reconstruction process. All images were saved in GIF format for off line processing and analysis.

\subsection{Image Analysis}

In this study, image quality of reconstructed images was analyzed visually, by looking at the shape of hot and cold regions and their detectability. From quantitative analysis point of view, contrast of both regions was measured. For qualitative and quantitative analysis, 53rd and 42nd transverse image slices of hot and cold regions were chosen, respectively, based upon the visual examination of all reconstructed transverse image slices. In this regard, three researchers having more than five years work experience in the department of nuclear medicine imaging were engaged. Also the hot and cold region images were retrieved in an ImageJ (Version 1.50f - an open source image processing software) [16] environment for image contour analysis. Moreover, all individual images were displayed by selecting the similar image display parameters to avoid any inconsistency. As well as, more than one image in a single window was displayed for easy comparison and analysis purposes.

Hot regions' contrast was measured by applying Equation (2). Regions of interest (ROIs) were drawn using the software available with the imaging system mentioned earlier in the materials and methods section. Several circular shaped ROIs (according to the size of the region) were drawn and placed inside the hot region image to record the count density $\left(D_{\text {region }}\right)$. A large irregular ROI was drawn for measuring the background count density $\left(D_{b k g}\right)$; care was taken to avoid the overlapping of hot regions. All ROIs were copied and pasted onto the hot regions of next transverse image slice to maintain the uniformity.

$$
C_{H R}=\frac{D_{\text {region }}-D_{b k g}}{D_{\text {region }}+D_{b k g}}
$$

Contrast of cold regions was calculated using Equation (3). Regions of interest were drawn similarly as the hot regions contrast measurement.

$$
C_{C R}=\frac{D_{\text {region }}-D_{b k g}}{D_{b k g}}
$$

where $D_{\text {region }}$ is the mean count density in the hot and/or cold region and $D_{b k g}$ is the average count value in the adjacent area or the background area of the hot and/or cold region. 


\subsection{Statistical Analysis}

All measured values of contrast of hot and cold regions obtained from images reconstructed with a 0.35 cycles $\cdot \mathrm{cm}^{-1}$ and a 0.45 cycles $\cdot \mathrm{cm}^{-1}$ cut-off frequency and an order 5 were repeated for three times each. Oneway ANOVA test of significance $(p<0.05)$ was used to assess the difference between the contrast values.

\section{Results}

The goal of SPECT is to obtain a better image quality for accurate diagnosis of the disease. This study investigated the influence of varying cut-off frequency with a constant value of order of Butterworth filter on hot \& cold regions of different sizes at various locations into the phantom with similar transverse views as shown in Figure 2. Reconstructed images were analyzed visually, i.e., the regions' detectability, distortion of region's shape (contour analysis) and quantitatively by measuring the hot and cold regions contrast.

\subsection{Visual (Contour) Analysis of Hot Regions Image}

Figure 3, show transverse image slices of hot regions insert with two different cut-off frequencies, e.g., 0.35 and 0.45 cycles $\cdot \mathrm{cm}^{-1}$ of the same order, i.e., 5, respectively. From Figure 3(a) three hot region pairs of diameter,

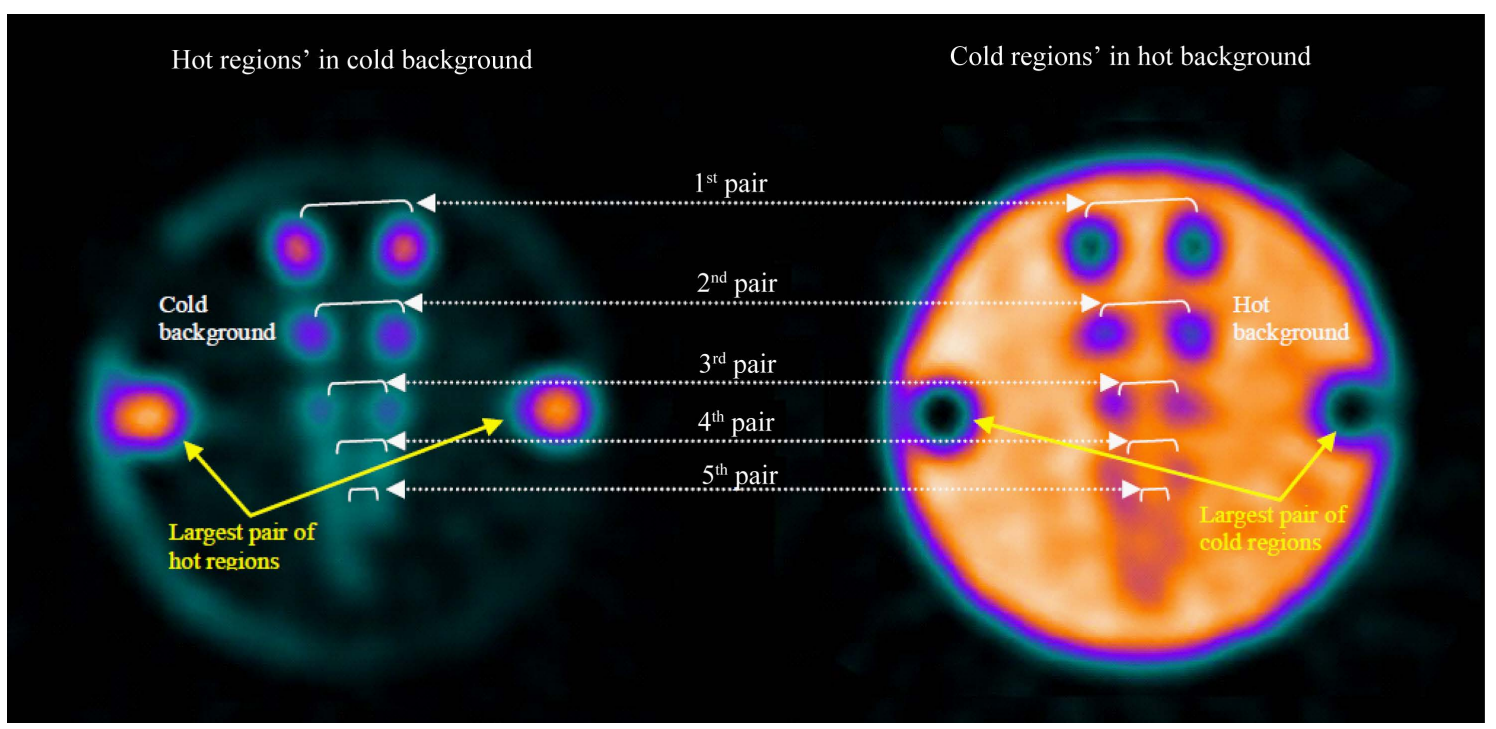

Figure 2. Show the details of hot and cold regions in terms of shape, pairs and positions. Two pairs (6th and 7th) are not shown in figure.

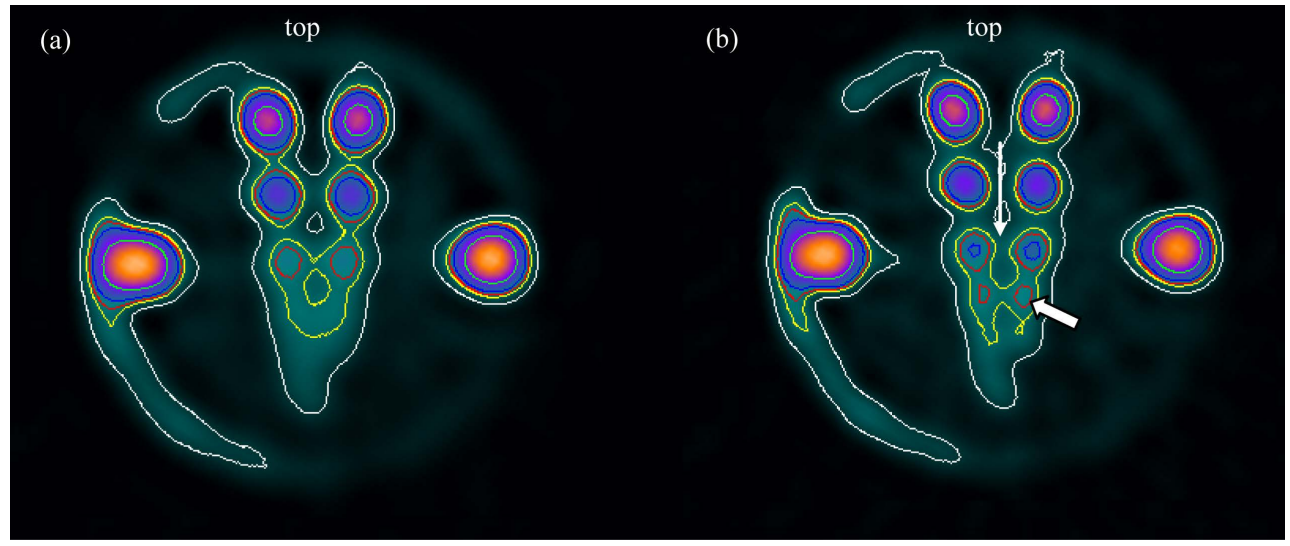

Figure 3. Transverse images of hot regions insert with (a) 0.35 cycles $\cdot \mathrm{cm}^{-1}$ and (b) $0.45 \mathrm{cycles} \cdot \mathrm{cm}^{-1}$ cut-off frequencies and an order 5 of filter. 
$22.6 \mathrm{~mm}, 18.1 \mathrm{~mm}$ and $14.5 \mathrm{~mm}$ can be seen clearly (excluding the largest pair) and the presence of a fourth pair in the image can be noticed. As for as concerns the shape of hot regions an almost circular shape of the first two pairs (top) of hot regions can be observed as compared to the third pair. Furthermore, there is less blurring around the hot region's pairs of diameter $22.6 \mathrm{~mm}$ and $18.1 \mathrm{~mm}$ thus, the gap between them can be seen more clearly. However, the space between the other smaller hot regions cannot be observed, due to the presence of noise.

Figure 3(b) illustrates a transverse image slice of hot regions obtained with a $0.45 \mathrm{cycles} \cdot \mathrm{cm}^{-1}$ cut-off frequency of order 5. In this case, four pairs - fourth pair indicated by thick white arrow (excluding the largest pair) of hot regions of diameter $22.6 \mathrm{~mm}, 18.1 \mathrm{~mm}, 14.5 \mathrm{~mm}$ and $11.6 \mathrm{~mm}$ are visible with an eyeball's judgment compared to the image produced using a 0.35 cycles $\mathrm{cm}^{-1}$ cut-off frequency. Moreover, hot regions in the image obtained by using higher cut-off frequencies are also in a circular shape, sharper and clearer $(18.1,14.5$ and $11.6 \mathrm{~mm}$ diameter) compared to the image shown in Figure 3(a). However, the fourth pair is blurry and connected as shown in Figure 3(b).

Furthermore, the overall image quality is improved; three pairs of hot regions are seen separately (space between third pair is shown by thin white arrow) compared to the 0.35 cycles $\cdot \mathrm{cm}^{-1}$ cut-off frequency image, though there is appearance of noise in the background. In addition to that, the location of the fifth pair can be identified which reflect the enhancement in the region detectability. Remaining smaller hot regions are undetectable.

\subsection{Hot Regions Contrast Analysis}

For contrast measurement three pairs from images shown in Figure 3(a) and Figure 3(b) were included because of their clarity and the ease in fixing the ROI inside the hot region. However, it was difficult to place the ROI on the fourth pair due to unclear edges. Therefore it was excluded from contrast calculations. In addition to that, contrast of only one hot region of the pair was measured because of the same size.

Figure 4 shows the hot regions image contrast when $0.35 \mathrm{cycles} \cdot \mathrm{cm}^{-1}$ cut-off frequency was applied and the contrast values for hot regions of diameter $22.6 \mathrm{~mm}, 18.1 \mathrm{~mm}$ and $14.5 \mathrm{~mm}$ were $81 \%, 85 \%$, and $71 \%$, respectively. Whereas, the measured contrast values for hot regions of diameter $22.6 \mathrm{~mm}, 18.1 \mathrm{~mm}$ and $14.5 \mathrm{~mm}$ with 0.45 cycles $\cdot \mathrm{cm}^{-1}$ cut-off frequency were $82 \%, 87 \%$, and $72 \%$, respectively. Generally, marginal improvement in contrast with the increase of cut off frequency has been achieved, i.e., selecting a 0.45 cycles $\mathrm{cm}^{-1}$ cut-off frequency as compared to a $0.35 \mathrm{cycles} \cdot \mathrm{cm}^{-1}$ cut-off frequency.

\subsection{Visual (Contour) Analysis of Cold Regions Image}

The effects of cut-off frequency of the Butterworth filter on cold region images as shown in Figure $\mathbf{5}$ were investigated using visual analysis.

It can clearly be seen that image in Figure 5(a) reconstructed with a low cut-off frequency $\left(0.35 \mathrm{cycles} \cdot \mathrm{cm}^{-1}\right)$

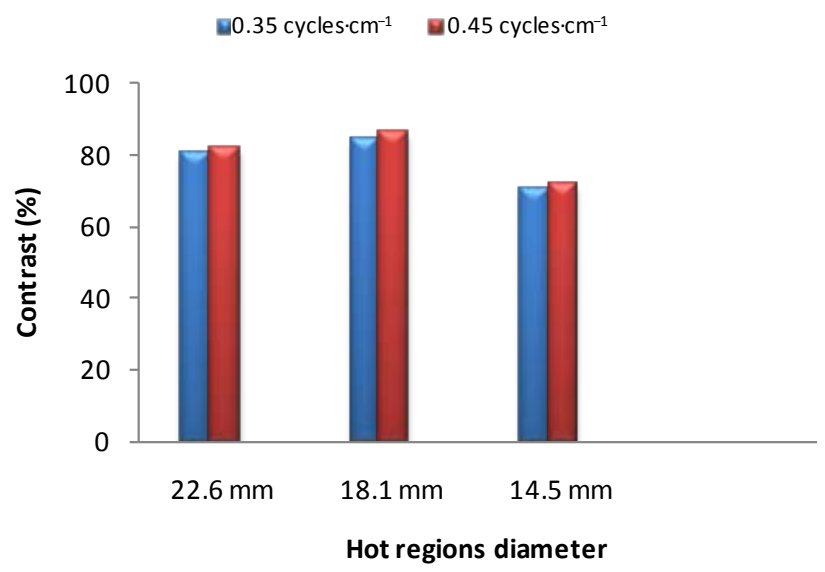

Figure 4. Contrast of hot regions of different diameter with different cut-off frequency values. 


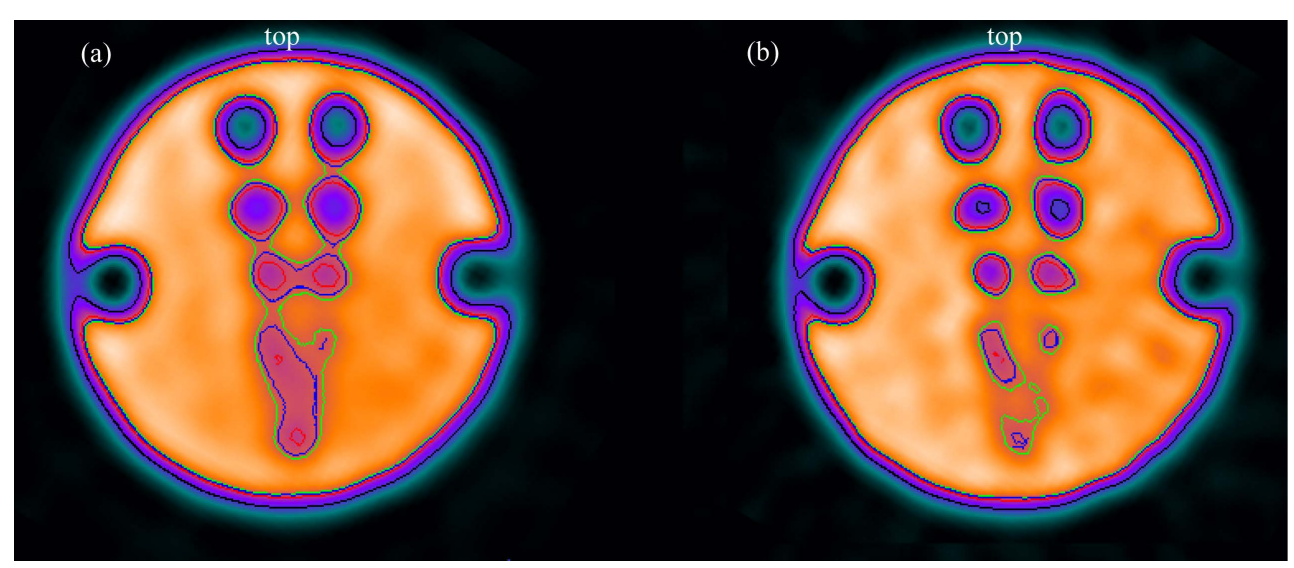

Figure 5. Transverse images of cold regions insert with (a) 0.35 cycles $\cdot \mathrm{cm}^{-1}$ and (b) 0.45 cycles $\cdot \mathrm{cm}^{-1}$ filter cut-off frequencies and an order 5.

is smoother than that from the high cut-off frequency $\left(0.45 \mathrm{cycles} \cdot \mathrm{cm}^{-1}\right)$ image presented in Figure 5(b). Also, fewer cold region pairs (three pairs from top-excluding the largest pair) of size $22.5 \mathrm{~mm}, 18.0 \mathrm{~mm}$ and $14.4 \mathrm{~mm}$ diameter can be viewed when a low cut-off frequency filter was selected. In the image shown in Figure 5(b) there are three clearly spaced and detectable cold region pairs $(22.5 \mathrm{~mm}, 18.0 \mathrm{~mm}$ and $14.4 \mathrm{~mm}$ diameter $)$ and one region of the fourth pair detached from the other fellow region, i.e., $11.5 \mathrm{~mm}$ diameter (the largest pair situated at the sides opposite to each other at $180^{\circ}$ is excluded). Moreover, the gap between each cold region pair reflects the less background noise in image as shown in Figure 5(b) relative to the image depicted in Figure 5(a). However, the shape of detectable cold regions appears oval as compared to image in Figure 5(a). Concerning the improvement in detectability of cold regions pairs, more pairs can be viewed in a reconstructed image when applying a $0.45 \mathrm{cycles} \cdot \mathrm{cm}^{-1}$ cut-off frequency as compared to $0.35 \mathrm{cycles} \cdot \mathrm{cm}^{-1}$ cut-off frequency.

\subsection{Cold Regions Contrast Analysis}

For contrast measurement only two pairs of cold regions from the image shown in Figure 5(a) and three pairs from the image shown in Figure 5(b) were included because of their sharper boundaries compared to those of the remaining cold regions. In this case the largest cold region pair was not included. Figure 6 shows the contrast values of cold regions (all contrast values are measured as negative but they have been presented in the graph as positive for the sake of simplicity).

The measured contrast of cold regions from the image that was reconstructed using 0.35 cycles $\mathrm{cm}^{-1}$ cut-off frequency sized, $22.5 \mathrm{~mm}$ and $18.0 \mathrm{~mm}$ diameter is $56 \%$ and $41 \%$, respectively. However, contrast of cold regions calculated from the image obtained by applying a $0.45 \mathrm{cycles} \cdot \mathrm{cm}^{-1}$ cut-off frequency was $65 \%, 46 \%$ and $32 \%$ for cold regions of diameter $22.5 \mathrm{~mm}, 18.0 \mathrm{~mm}$ and $14.4 \mathrm{~mm}$, respectively. A significant improvement was achieved in the contrast of cold region images when a higher cut-off frequency was applied during the image reconstruction process.

\section{Discussion}

The choice of filter and selection of its functions is generally subjective and investigational. There is no single optimized/standardized filter that can be used for all routine SPECT clinical examinations. In addition, the selection of filter in SPECT reconstruction depends on many factors, for example, type of patient study (type of organ), energy of gamma ray photon, image count density, dose administered into the patient, statistical noise, amount of background noise, type of collimator and the information desired by a clinician as well as the preference of the interpreting physician in routine clinical studies [2] [7] [17]. Thus, it is difficult to select a filter and its functions as a "filter of choice".

The most important objectives of filtering the SPECT raw data are to suppress noise, improve image quality, enhance the edges of target organ and recover image resolution. Consequently, accuracy in the diagnosis of diseases is achieved and therefore, best patient care can be provided. Our study explored the effects on detectability, shape and image quality of hot and cold regions' pairs with different sizes and at different locations in the phan- 


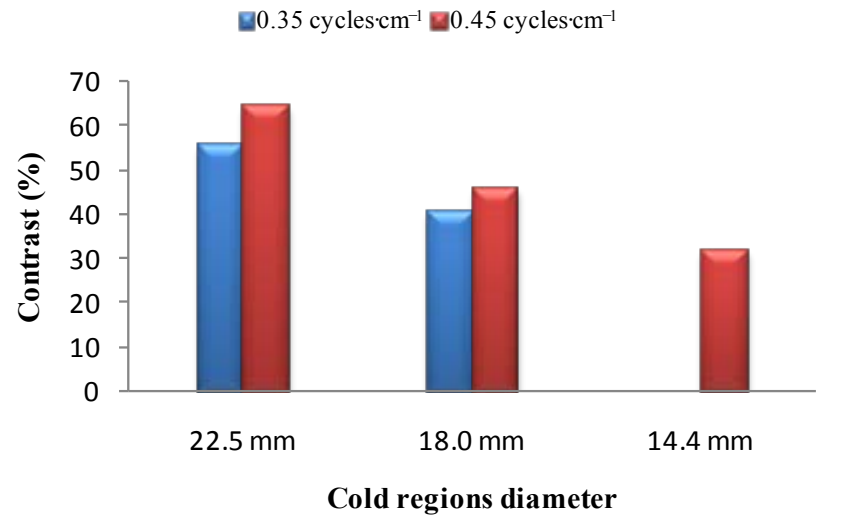

Figure 6. Contrast of cold regions of different diameters with different cut-off frequency values.

tom by choosing different cut-off frequency values of Butterworth filter. The reason for the selection of this filter is that it is most commonly used in SPECT imaging. It is imperative to mention that the design of the cold and hot regions' insert provide similar transverse views of both types of regions which facilitates the analysis of images and uncover any differences in an image quality that could have occurred due to the change of cut-off frequency.

Effects of changing filter cut-off frequency have been examined by the visual interpretation of transverse image slices of hot regions, Figure 3(a) and Figure 3(b) were reconstructed by selecting a $0.35 \mathrm{cycles} \cdot \mathrm{cm}^{-1}$ and a 0.45 cycles $\cdot \mathrm{cm}^{-1}$ cut-off frequency, respectively. It has been found that images with a high cut-off frequency show more of hot region pairs and are also sharper evidencing the improvement in the region's detectability. For hot regions, higher frequency component in the image can be corresponded with higher radioactivity distribution. Thus, the Butterworth filter allowed passing the frequency that matched to a $0.45 \mathrm{cycles} \cdot \mathrm{cm}^{-1}$ and suppressed the other frequency components which are assumed as noisy signals. On the other hand, the lower the cut-off frequency, the smoother the image, i.e., better appearance as shown in Figure 3(a). However, a loss of resolution occurred in the image.

The cold region images shown in Figure 5(a) and Figure 5(b) are also investigated in terms of regions detectability and shape which were produced by using a $0.35 \mathrm{cycles} \cdot \mathrm{cm}^{-1}$ and a $0.45 \mathrm{cycles} \cdot \mathrm{cm}^{-1}$ cut-off frequency, respectively. Our results witness that with a higher cut-off frequency, i.e., $0.45 \mathrm{cycles}^{\cdot} \mathrm{cm}^{-1}$ four cold region pairs (the largest pair located at the sides opposite to each other at $180^{\circ}$ is not included) are viewed and spaced clearly. It has been reported that cold area is responsible for higher frequencies in the image data, i.e. rapid changes in the count density [18] [19]. From our findings, this holds true concerning the relevance of higher frequencies with the cold regions image. However, the drawback of using a high cut-off frequency is distortion of the shape of cold region (except the first pair from the top) which are in fact circular. Thus, in patient studies this effect would lead to misdiagnosis of the disease. Moreover, the higher cut-off frequency produces little grainy background in the image. The use of higher frequency filter retains the high frequency signals in the image thus it appears rougher [7].

Furthermore, results show that, more hot regions were detected compared to cold regions, when a 0.45 cycles $\mathrm{cm}^{-1}$ cut-off frequency was selected in the reconstruction process. Thus, the region detectability is better for hot regions relative to cold regions. In addition to that, it has been observed that higher cut-off frequency of Butterworth filter has the ability to detect smaller hot and cold regions than low cut-off frequency Figure 3(b) and Figure 5(b), respectively.

The analysis of contrast results of hot regions showed marginal improvement with the use of a higher cut-off frequency $\left(0.45\right.$ cycles $\left.\cdot \mathrm{cm}^{-1}\right)$. Overall, only a $2 \%$ to $3 \%$ increase in the contrast was achieved for those hot regions which were included in the contrast analysis. However, significant improvement $(p<0.05)$ in the contrast of $22.5 \mathrm{~mm}$ diameter cold region and conversely insignificant improvement $(\mathrm{p}>0.05)$ of $18.0 \mathrm{~mm}$ diameter cold region with the use of a higher cut-off frequency relative to a lower cut-off frequency is achieved. This is due to the fact that, the frequency in image data is target organ dependent and if the target organs are different from each other (as in this case) and thus their image frequency characteristics too [2]. Furthermore, hot region con- 
trast values are higher with both values of cut-off frequency relative to cold regions. However, in terms of improvement obtained in contrast of cold regions fared better than hot regions.

\section{Conclusion}

Effects of Butterworth filter functions were investigated on transverse images of both hot and cold regions of similar view. It was found that, a 0.45 cycles $\mathrm{cm}^{-1}$ cut-off frequency provides better results in terms of detectability for both types of regions i.e., hot and cold regions as well as high contrast values relative to a 0.35 cycles $\cdot \mathrm{cm}^{-1}$ cut-off frequency. However, with a 0.45 cycles $\cdot \mathrm{cm}^{-1}$ cut-off frequency the shape of cold regions was distorted. Our results confirmed that effects on image quality are different with the change of cut-off frequency which alters the image quality and thereby affecting quantitative analysis. Thus, prior to reconstruction of images, care should be taken in selecting a filter cut-off frequency, especially, when both types of regions (hot and cold) in the reconstructed image of a scanned object are expected.

\section{Acknowledgements}

Authors are thankful to the Department of Nuclear Medicine, Oncology and Radiotherapy, School of Medical Sciences, Universiti Sains Malaysia, Health Campus, 16150 Kubang Kerian, Kota Bharu, Kelantan, Malaysia for permitting experiments to be carried out for this research work and their staff for its cooperation and technical help. We acknowledge the help rendered by Syed Bilal Shah, Faculty of Engineering, and International Islamic University Malaysia in understanding the use of ImageJ software.

\section{References}

[1] Tusi, B.M.W. (1996) The AAPM/RSNA Physics Tutorials for Resident. Radiographics, 16, 173-183. http://dx.doi.org/10.1148/radiographics.16.1.173

[2] van Laere, K., Koole, M., Lemahieu, I. and Dierckx, R. (2001) Image Filtering in Single-Photon Emission Computed Tomography: Principles and Applications. Computerized Medical Imaging and Graphics, 25, 127-133. http://dx.doi.org/10.1016/S0895-6111(00)00063-X

[3] Heller, G.V., Mann, A. and Hendel, R.C. (2009) Nuclear Cardiology: Technical Applications. McGraw-Hill, New York.

[4] Sadremomtaz, A. and Taherparvar, P. (2013) The Influence of Filters on the SPECT Image of Carlson Phantom. Journal of Biomedical Science and Engineering, 6, 291-297. http://dx.doi.org/10.4236/jbise.2013.63037

[5] Lyra, M., Ploussi, A., Rouchota, M. and Synefia, S. (2014) Filters in 2D and 3D Cardiac SPECT Image Processing. International Journal of Biomedical Imaging, 2014, Article ID: 963264.

[6] Lyra, M. and Ploussi, A. (2011) Filtering in SPECT Image Reconstruction. International Journal of Biomedical Imaging, 2011, Article ID: 693795. http://dx.doi.org/10.1155/2011/693795

[7] Pandey, A.K., Pant, G.S. and Malhotra, A. (2004) Standardization of SPECT Filter Parameters. Indian Journal of Nuclear Medicine, 19, 30-35.

[8] Christian, P.E., Bernier, D.R. and Langan, J.K. (2003) Nuclear Medicine and PET: Technology and Techniques. 5th Edition, Elsevier, Mosby.

[9] Miller, T.R. and Sampathkumaran, K.S. (1982) Digital Filtering in Nuclear Medicine. Indian Journal of Nuclear Medicine, 23, 66-72.

[10] Reijo, T., Heli, H., Heimo, I. and Antti, S. (2015) Adaptive Autoregressive Model for Reduction of Noise in SPECT. Computational and Mathematical Methods in Medicine, 2015, Article ID: 494691.

[11] Salar, S., Alireza, K.A., Mohammad, R.A., Mohammad, H.F. and Arman, R. (2013) A Novel Non-Linear Recursive Filter Design for Extracting High Rate Pulse Features in Nuclear Medicine Imaging and Spectroscopy. Medical Engineering \& Physics, 35, 754-764. http://dx.doi.org/10.1016/j.medengphy.2012.08.003

[12] Duarte, D.D., Monteiro, M.S., El Hakmaoui, F., Prior, J.O., Vierira, L. and Pires-Jorge, J. (2012) Influence of Reconstruction Parameters during Filtered Backprojection and Ordered-Subset Expectation Maximization in the Measurement of the Left-Ventricular Volumes and Function during Gated SPECT. Journal of Nuclear Medicine Technology, 40, 29-36. http://dx.doi.org/10.2967/jnmt.111.094599

[13] Sayed, I.S. and Shah, A.A. (2007) Modified PET/SPECT Cylindrical Phantom. IFMBE Proceedings of the World Congress on Medical Physics and Biomedical Engineering, WC2006, Seoul, 27 August-1 September 2006, 1681-1683. http://dx.doi.org/10.1007/978-3-540-36841-0_414 
[14] Zeniya, T., Watabe, H., Aoi, T., Kyeong, M., Teramoto, N., Hayashi, T., Sohlberg, A., Kudo, H. and Iida, H. (2004) A New Reconstruction Strategy for Image Improvement in Pinhole SPECT. European Journal of Nuclear Medicine and Molecular Imaging, 31, 1166-1172. http://dx.doi.org/10.1007/s00259-004-1510-4

[15] Sayed, I.S. and Aima, H.H. (2011) Evaluation of the Effect of Chang's Attenuation Correction Technique on Similar Transverse Views of Cold and Hot Regions in Tc-99m SPECT: A Phantom Study. IFMBE Proceedings of 5th Kuala Lumpur International Conference on Biomedical Engineering, BIOMED 2011, Kuala Lumpur, 20-23 June 2011, 643649. http://dx.doi.org/10.1007/978-3-642-21729-6_158

[16] Rasband, W.S. (1997-2015) ImageJ. National Institutes of Health, Bethesda, Maryland, USA. http://imagej.nih.gov/ij

[17] Onishi, H., Matsutake, Y., Matsutomo, N. and Amijima, H. (2010) Validation of Optimal Cut-Off Frequency Using a Butterworth Filter in Single Photon Emission Computed Tomography Reconstruction for the Target Organ: Spatial Domain and Frequency Domain. Humanity and Science: Journal of the Faculty of Health and Welfare, Prefectural University of Hiroshima, 10, 27-36.

[18] Takavar, A., Shamsipour, G., Sohrabi, M. and Eftekhari, M. (2004) Determination of Optimum Filter in Myocardial SPECT: A Phantom Study. International Journal of Radiation Research, 4, 205-210.

[19] Lima, A.L.S., de Jesus, M.C., dos Santos, J.A. and Megueriam, B.A. (2007) Influence of Digital Filters Used to Obtain Cerebral Image in Nuclear Medicine. Proceeding of International Nuclear Atlantic Conference-INAC 2007, Santos, 30 September-5 October 2007, 5. https://www.ipen.br/biblioteca/cd/inac/2007/pdf_dvd/E05 1221.pdf 